\title{
Measurement of the Properties of the Higgs Boson at ATLAS
}

\author{
Timothy Bristow, on behalf of the ATLAS Collaboration \\ SUPA - School of Physics and Astronomy, University of Edinburgh, Edinburgh, United Kingdom
}

\begin{abstract}
An update on the Higgs boson search in the decay channels $H \rightarrow \gamma \gamma, H \rightarrow Z Z^{(*)} \rightarrow 4 l, H \rightarrow W W^{(*)} \rightarrow l v l v$, $H \rightarrow \tau^{+} \tau^{-}$and $H \rightarrow b \bar{b}$ at the ATLAS detector is presented. Proton-proton collision data recorded by the ATLAS experiment corresponding to an integrated luminosity of up to $25 / \mathrm{fb}$ at centre-of-mass energies of 7 and $8 \mathrm{TeV}$ are used for these results. The latest combined and individual channel measurements of the mass, signal strength, spin and parity, coupling constants and Higgs boson production are reported. Results on the measurements of the properties of the Higgs boson are all consistent with the Standard Model.
\end{abstract}

Keywords: ATLAS, LHC, Higgs, Standard Model

PACS: 14.80.Bn, 13.38.-b, 11.30.Er, 13.85.Rm

\section{INTRODUCTION}

The Standard Model (SM) describes physics on a subatomic scale, including the interactions and fundamental particles. The Brout-Englert-Higgs mechanism is required to give masses to the $W$ and $Z$ bosons and the fermions through electroweak symmetry breaking $[1,2,3,4]$ by introducing a complex scalar field. This Higgs field interacts with particles giving them mass. The excitations of this field introduce a new particle: the Higgs boson. Over the last four decades the SM has been tested by experiment and has shown very good agreement with data for high energy particle interactions. However, until recently the Higgs mechanism and the associated Higgs boson were not experimentally verified. A new boson was discovered by the ATLAS and CMS collaborations at CERN [5] and announced on the 4th of July 2012 that is compatible with a SM Higgs boson at a mass of $\approx 125 \mathrm{GeV}$.

This note gives an update on the latest results for the mass, signal strength, spin and parity and coupling constants for the Higgs boson search. Higgs boson production and decay channels are described in Section 2. A short description of the ATLAS detector is given in Section 3. The latest measurements on the signal mass and signal strength (Section 4) are divided into bosonic ${ }^{1}$ (Section 4.1) and fermionic decay channels ${ }^{2}$ (Section 4.2). The spin and parity measurments are presented in Section 5. Finally, the results of the coupling measurements are given in Section 6.

\section{HIGGS BOSON PRODUCTION AND DECAY AT THE LHC}

The production of the SM Higgs boson at the LHC is most likely to occur through gluon fusion (ggF), vector boson fusion (VBF) and Higgs-Strahlung with an associated vector boson [6] ( $V H$, where $V$ denotes a $W$ or $Z$ boson), or top fusion $(t t H)$, see Fig. 1a-d. Gluon fusion offers a higher production cross section than vector boson fusion by an order of magnitude, but there is also a large QCD background.

Higgs boson decays can proceed through either fermionic or bosonic decay channels. The major discovery channels are the bosonic channels $H \rightarrow \gamma \gamma, H \rightarrow Z Z^{(*)} \rightarrow 4 l$ and $H \rightarrow W W^{(*)} \rightarrow l v l v$. Here the $l$ refers to either $e$ or $\mu$. The largest and most sensitive fermionic decays are $H \rightarrow \tau^{+} \tau^{-}$and $H \rightarrow b \bar{b}$. The branching ratios are determined by the mass of the Higgs boson (see Fig. 1e).

\footnotetext{
${ }^{1}$ Bosonic decay channels presented are $H \rightarrow \gamma \gamma, H \rightarrow Z Z^{(*)} \rightarrow 4 l$ and $H \rightarrow W W^{(*)} \rightarrow l v l v$

${ }^{2}$ Fermionic decay channels presented are $H \rightarrow \tau^{+} \tau^{-}$and $H \rightarrow b \bar{b}$.
} 
(a)

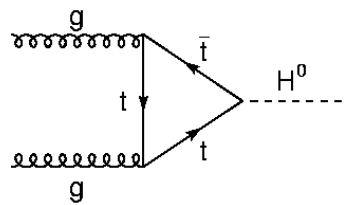

(c)

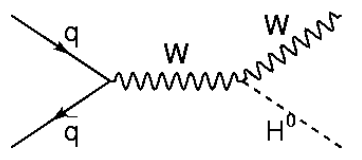

(a)-(d) (b)

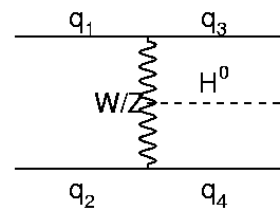

(d)

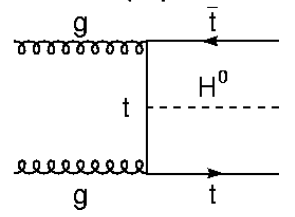

g

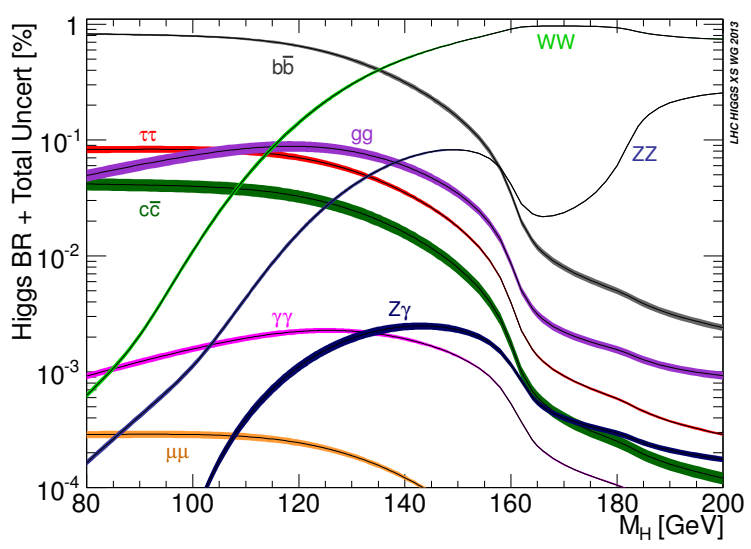

(e)

FIGURE 1. The different Higgs production mechanisms are shown in (a)-(d) and the branching ratios for a low mass Standard Model Higgs boson in (e)[7]. The favoured branching ratio at a mass of $m_{H}=125 \mathrm{GeV}$ is to two $b$ quarks, however, the bosonic channels, $W W, Z Z$ and $\gamma \gamma$, have much lower backgrounds.

\section{THE ATLAS DETECTOR}

The Large Hadron Collider (LHC)[8] is a proton-proton particle accelerator with a design centre-of-mass energy of $\sqrt{s}=14 \mathrm{TeV}^{3}$ at CERN near Geneva on the border of Switzerland and France. Two proton beams are accelerated in opposing directions in a $27 \mathrm{~km}$ circular tunnel $\approx 100 \mathrm{~m}$ underground. These are collided at multiple points around the ring within the different detectors.

The ATLAS detector[9] is the largest particle detector at the LHC measuring $45 \mathrm{~m}$ in length and $25 \mathrm{~m}$ in diameter. It is a multipurpose detector with forward-backward cylindrical symmetry divided into four main subsystems. The inner tracking detector (ID) measures charged particle position and momentum. This consists of a silicon pixel detector, semi-conductor tracker (SCT) which uses silicon strips and the transition radiation tracker (TRT) which cover the pseudorapidity ${ }^{4}$ ranges $|\eta|<2.5$ and $|\eta|<2.0$, respectively. The pixel detector has 1744 modules with about 80 million readout channels and the SCT has 4088 modules with about 6 million readout channels.

The ID is surrounded by a $2 \mathrm{~T}$ solenoidal superconducting magnet and the calorimeters. The electromagnetic sampling calorimeter (EM) uses liquid-argon (LAr) as the sampling material, covering pseudorapidities $|\eta|<1.475$ in the barrel regions and $1.375<|\eta|<3.5$ in the endcap regions. The hadronic calorimeter (HCal) surrounds the EM covering $|\eta|<4.9$. LAr and scintillating tiles are used as active sampling materials in the HCal. The muon spectrometer is the outermost subsystem, consisting of three air-core superconducting toroidal magnets each with eight superconducting coils, tracking chambers (covering $|\eta|<2.7)$ and trigger chambers $(|\eta|<2.4)$. The detector provides measurements up to $|\eta|<2.5$ for photons and leptons and $|\eta|<4.9$ for jets and missing transverse energy.

\section{MASS MEASUREMENTS AND SIGNAL STRENGTH}

The latest mass measurements and signal strengths are shown individually in the following sections with a combined result given at the end. The first subsection describes the $H \rightarrow \gamma \gamma, H \rightarrow Z Z^{(*)} \rightarrow 4 l$ and $H \rightarrow W W^{(*)} \rightarrow l v l v$ channels, which provided the most significance for the discovery of the Higgs boson. The subsequent subsection describes the $H \rightarrow \tau^{+} \tau^{-}$and $H \rightarrow b \bar{b}$ channels, where the sensitivity is lower due to the large backgrounds.

\footnotetext{
${ }^{3}$ During 2011 it was run at $\sqrt{s}=7 \mathrm{TeV}$ and during 2012 at $\sqrt{s}=8 \mathrm{TeV}$.

4 A right-handed coordinate system is used at ATLAS, with the origin in the centre of the detector. The axes are $z$ along the beam line, $y$ upwards and $x$ towards the centre of the LHC ring. Pseudorapidity is defined as $\eta=-\ln \tan (\theta / 2)$ where $\theta$ is the polar angle.
} 


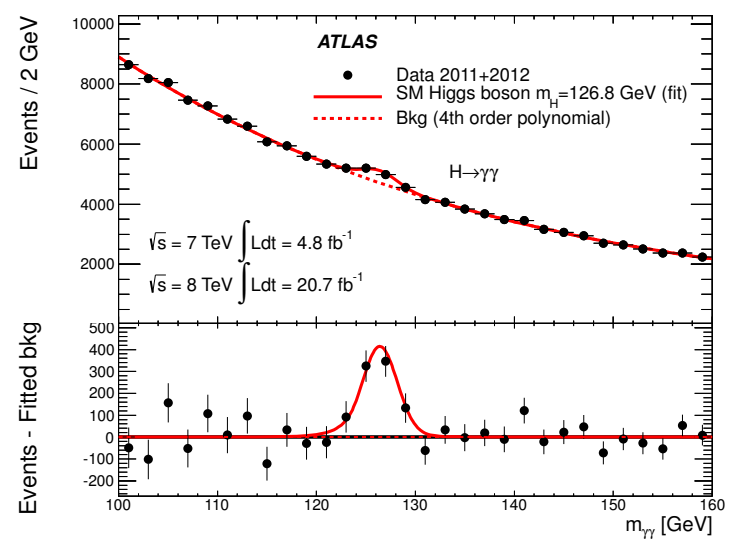

(a)

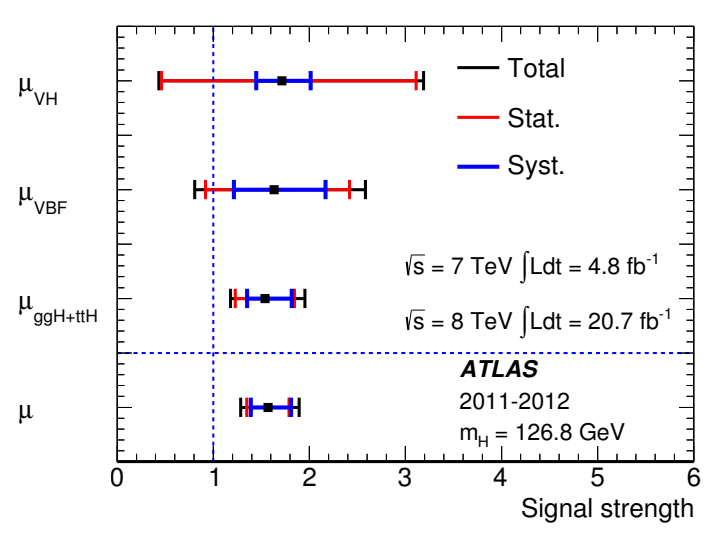

(b)

FIGURE 2. The $H \rightarrow \gamma \gamma$ invariant mass distribution is shown in (a)[13]. An excess with a local significance of $7.4 \sigma$ is seen at a mass of $m_{H}=126.8 \pm 0.2$ (stat.) \pm 0.7 (syst.). The signal strengths for the separate production mechanisms, $V H, \mathrm{VBF}$ and $g g F+t t H$, and the combined diphoton signal strength are shown in (b). The combined signal strength is $\mu=1.65 \pm$ (stat. $)_{-0.18}^{+0.25}$ (syst.) [13].

\subsection{Bosonic Decay Channels}

$$
\text { 4.1.1. } H \rightarrow \gamma \gamma
$$

The $H \rightarrow \gamma \gamma$ decay channel results are obtained using the full data sample collected at $\sqrt{s}=8 \mathrm{TeV}$ in 2012 , corresponding to $20.7 \mathrm{fb}^{-1}$, and the full data sample from 2011 at $\sqrt{s} 7=\mathrm{TeV}$, corresponding to $4.8 \mathrm{fb}^{-1}$ [25]. A peak in the distribution of the invariant mass of two high- $p_{T}$ photons is searched for. The photons are selected using clusters of energy in the Electromagnetic (EM) calorimeter with requirements on the shower shapes. The photon candidates must be isolated and have a transverse energy greater than $40 \mathrm{GeV}$ and $30 \mathrm{GeV}$, respectively. The full details of the object and event reconstruction are given in [12]. The events are divided into 10 (14) exclusive categories for the 2011 (2012) data to maximise sensitivity, several of which are used to provide further sensitivity to the VBF and $\mathrm{VH}$ production modes.

The branching ratio for this channel is relatively low with a large irreducible background from $q \bar{q}+g g \rightarrow \gamma \gamma$ continuum production, and a reducible background from $\gamma+$ jet and jet-jet production where the jets are misidentified as photons. To provide a handle on the backgrounds the invariant mass distribution is parameterised with different analytic functions. The parameters are set using fits to the data for diphoton events. This is done separately for each event category. The function used is determined by the event category and is either a fourth-order Bernstein polynomial, the exponential of a second-order polynomial or a single exponential. The expected number of signal events is modelled using simulated events. The smooth invariant mass distribution allows for a good determination of the background.

An excess of events is seen at a mass of $126.8 \pm 0.2$ (stat.) \pm 0.7 (syst.) (see Fig. 2a) with a local significance of $7.4 \sigma$. The VBF category alone has a local significance of $2.0 \sigma$. The signal strengths ${ }^{5}$ for the production mechanisms $V H$, VBF and ggF $+t t H$ are shown in Fig. $2 \mathrm{~b}$, with an overall combined signal strength of $\mu=1.65 \pm(\text { stat. })_{-0.18}^{+0.25}$ (syst.).

$$
\text { 4.1.2. } H \rightarrow Z Z^{(*)} \rightarrow 4 l
$$

The $H \rightarrow Z Z^{(*)} \rightarrow 4 l$ analysis uses $4.6 \mathrm{fb}^{-1}$ of data taken in 2011 at $\sqrt{s}=7 \mathrm{TeV}$ and $20.7 \mathrm{fb}^{-1}$ of data taken in 2012 at $\sqrt{s}=8 \mathrm{TeV}$ [18]. It provides sensitivity to a wide mass range from $110 \mathrm{GeV}$ to $600 \mathrm{GeV}$ where the invariant mass of the final four leptons is reconstructed and used as the discriminating variable. The states analysed are $4 e, 4 \mu$, $2 e 2 \mu$ and $2 \mu 2 e$, where the final two states here differ by having the first two leptons closest to the $Z$ pole mass. The

\footnotetext{
5 The signal strength, $\mu$, is defined as the ratio of the observed cross section to the Standard Model prediction, $\mu=\frac{\sigma}{\sigma_{S M}}$
} 


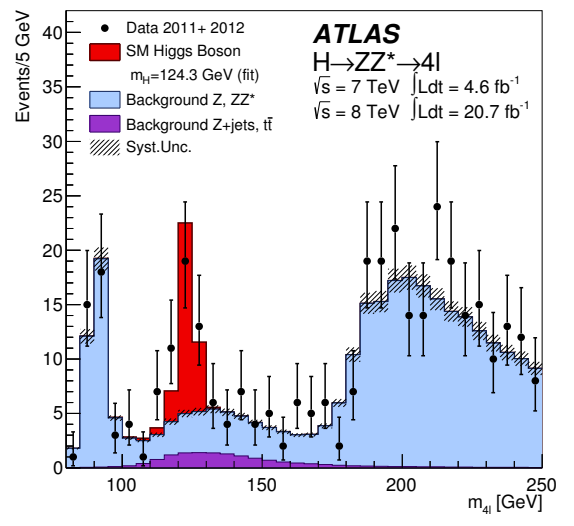

(a)

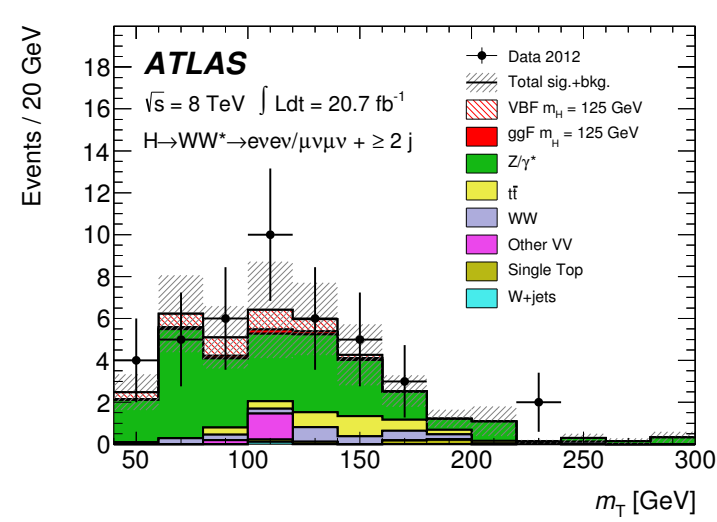

(b)

FIGURE 3. The $H \rightarrow Z Z^{(*)} \rightarrow 4 l$ invariant mass distribution is shown in (a)[18]. An excess with a local significance of $6.6 \sigma$ is seen at a mass of $124.3_{-0.5}^{+0.6}$ (stat. ${ }_{-0.3}^{+0.5}$ (syst.). Plot (b) shows the transverse mass distribution for $H \rightarrow W W \rightarrow l v l v$ for the case where the leptons are of the same flavour and there are 2 or more jets. The signal excess is clearly seen in plot (a) and the signal excess separated into VBF and ggF in plot (b)[13].

pair of leptons with an invariant mass closest to the $Z$ mass are required to have $50<m_{12}<106 \mathrm{GeV}$, and the other pair's invariant mass must be in the range $m_{\min }<m_{34}<115 \mathrm{GeV}$, where $m_{\min }$ varies according to the mass of all four leptons, $m_{4 l}$. To increase sensitivity and measure cross sections for the VBF, VH and ggF production mechanisms events are categorised by the presence of two forward jets, an additional lepton or the lack thereof.

The four final lepton states give a clean signature with a high signal to background ratio. The largest irreducible background comes from continuum $Z Z^{(*)}$ production which is modelled using MC simulation. Smaller reducible backgrounds come from $Z+$ jets and $t \bar{t}$ and are estimated using data-driven methods.

An excess is clearly observed at a mass of $124.3_{-0.5}^{+0.6}$ (stat. $)_{-0.3}^{+0.5}$ (syst.) $\mathrm{GeV}$ (Fig. 3a) with a local significance of $6.6 \sigma$. The best fit signal strength is found to be $\mu=1.7_{-0.4}^{+0.5}$. In addition, a VBF like candidate was observed at $123.5 \mathrm{GeV}$ with a signal to background ratio of 1 .

$$
\text { 4.1.3. } H \rightarrow W W^{(*)} \rightarrow l v l v
$$

The $H \rightarrow W W^{(*)} \rightarrow l v l v$ analysis [26] is done using the same amount of data as the $H \rightarrow Z Z^{(*)} \rightarrow 4 l$ analysis. The invariant mass cannot be used as a discriminating variable due to the neutrinos in the $W$ decays. Instead, the key variables are the transverse mass, $m_{T}^{6}$, and the invariant mass of the two leptons, $m_{l l}$. To increase sensitivity, events are categorised according to the number of jets $(0,1$ or $2+)$ in the event and the flavour of the leptons, evev, ev $\mu v$ and $\mu \nu \mu v$. The categorisation based on the number of jets provides sensitivity to the production mode, specifically, the inclusion of the 2 jet category provides VBF production measurements. Distributions of $m_{T}$ for 2-jet events, with the different ggF and VBF contributions, are shown in Fig. $3 \mathrm{~b}$.

Events are selected using different criteria for each category after applying a similar preselection. The missing transverse energy, $E_{T}^{\text {miss }}$, and the missing transverse momentum, $p_{T}$, and the angle between them and between the two leptons are used to suppress Drell-Yan and multi-jet events. Further backgrounds due to $W W$ events, top quark production, $Z / \gamma^{*} \rightarrow \tau \tau / l l$ and $W / Z+$ jets are also present. The backgrounds are estimated using data-driven methods using simulations for corrections and normalisation.

A signal significance is seen at $m_{H}=125 \mathrm{GeV}$ of $3.8 \sigma$ (3.7 expected). The VBF channel ( 2 or more jets) has a significance of $2.5 \sigma$ (1.6 expected). The signal strength at this mass point is $\mu=1.01 \pm 0.31$.

\footnotetext{
${ }^{6}$ The transverse mass is given by $m_{\mathrm{T}}=\sqrt{2 p_{\mathrm{T}}^{\text {lep }} E_{T}^{\text {miss }}(1-\cos \Delta \phi)}$, where $\Delta \phi$ is the absolute value of the difference between the $\phi$ of the lepton and missing energy.
} 


\subsubsection{Combined Mass Results}

There is a slight mass discrepancy between the $H \rightarrow \gamma \gamma$ and $H \rightarrow Z Z^{(*)} \rightarrow 4 l$ channels. The compatibility between the mass measurements from the two channels is quantified using a combination of likelihood functions. First, the two masses, $m_{H}^{\gamma \gamma}$ and $m_{H}^{4 l}$, are varied independently. This is followed by a likelihood function for the mass difference $\Delta m_{H}=m_{H}^{\gamma \gamma}-m_{H}^{4 l}$. The estimated mass difference is calculated to be $\Delta m_{H}=2.3_{-0.7}^{+0.6}$ (stat.) \pm 0.6 (syst.) GeV. Evaluating the likelihood function at $\Delta m_{H}=0$ gives a probability for the observed $\Delta m_{H}$ of $1.2 \%$, or $1.5 \%(2.4 \sigma)$ using Monte Carlo ensemble tests. By using more conservative methods and incorporating uncertainties from $Z \rightarrow e e$ calibration, material upstream of the EM calorimeter and the energy scale in the presampler this compatibility is increased. The probability for the observed value to be found, disfavouring $\Delta m_{H}=0$, is increased to $8 \%$.

To combine the channels a profile likelihood ratio as a function of $m_{H}$ is used and the signal strength is allowed to vary in both channels. The combined mass is found to be $m_{H}=125.5 \pm 0.2$ (stat. $)_{-0.6}^{+0.5}$ (syst.) GeV [13], see Fig. 4a.

\subsection{Fermionic Decay Channels}

$$
\text { 4.2.1. } H \rightarrow \tau^{+} \tau^{-}
$$

The $H \rightarrow \tau^{+} \tau^{-}$analysis is done using $4.6 \mathrm{fb}^{-1}$ of 2011 data at $7 \mathrm{TeV}$ and $13.0 \mathrm{fb}^{-1}$ of 2012 data at $8 \mathrm{TeV}$ [20]. The $\tau$ can decay either leptonically (denoted $\tau_{\text {lep }}$ ) to an electron or muon and the corresponding neutrino, or it can decay hadronically (denoted $\tau_{\text {had }}$ ) into an odd number of charged pions, a $v_{\tau}$ and a number of neutral pions. The analysis is split into 12 (13) exclusive categories for the 8 (7) $\mathrm{TeV}$ data. The categories are chosen to separate the leptonic and hadronic decay modes, $H \rightarrow \tau_{\text {lep }} \tau_{\text {lep }}, H \rightarrow \tau_{\text {lep }} \tau_{\text {had }}$ and $H \rightarrow \tau_{\text {had }} \tau_{\text {had }}$, and to increase sensitivity to the different Higgs production mechanisms, VH, VBF and ggF, and to boosted Higgs production. The invariant mass $m_{\tau \tau}$ is reconstructed using the Missing Mass Calculator (MMC) [21] and used as the discriminating variable. The MMC uses the transverse momenta, missing transverse energy and the angle between the visible and missing transverse momenta.

There is a large irreducible background from $Z \rightarrow \tau^{+} \tau^{-}$events. This background is modelled using data-driven embedding techniques. $Z \rightarrow \mu^{+} \mu^{-}$events from data are used with the muons replaced by simulated $\tau$ s. The other background contributions from $Z \rightarrow l l+$ jets, diboson production and top quark production are simulated with Monte Carlo and the QCD multi-jet background is estimated with a data-driven method.

A 95\% confidence level (CL) on the observed cross section over the expected Standard Model cross section at $m_{H}=125$ is placed at 1.9 (expected 1.2). There is a nominal excess over background at this mass point corresponding to a local significance of $1.1 \sigma$ (1.7 expected). The overall signal strength observed is $\mu=0.7 \pm 0.7$.

$$
\text { 4.2.2. } V H \rightarrow V b \bar{b}
$$

This analysis is done using $4.6 \mathrm{fb}^{-1}$ of 2011 data at $7 \mathrm{TeV}$ and $20.3 \mathrm{fb}^{-1}$ of 2012 data at $8 \mathrm{TeV}$ [22]. The $H \rightarrow b \bar{b}$ has the largest branching ratio for a low mass Higgs boson, however, there is a very large background from QCD. This large background is reduced by requiring the presence of an additional $W$ or $Z$ boson. The final signature is a pair of high $p_{T} b$-jets and a lepton requirement from the decay of the vector boson. This can either be zero charged leptons where the vector boson is a $Z$ that decays to neutrinos, one charged lepton where the vector boson is a $W$ or two charged leptons where the $Z$ decays to two charged leptons. Additional constraints are placed on the missing transverse energy, the transverse momentum and the invariant mass of the two leptons. The invariant mass of the $b$-jet pair, $m_{b b}$, is used as the discriminating variable, characterised according to the $p_{T}$ of the vector boson.

The background composition is different for the different lepton categories. The zero lepton category has large contributions from $Z / W+$ jets and top quark processes, the one lepton category has contributions from $W+$ jets and top quark processes and the two lepton category receives major contributions from $Z+j$ ets. Most of the backgrounds are taken from simulation and normalised to data, except for the QCD multi-jet background which is estimated using data-driven methods.

There is no significant excess seen over the background and a $95 \% \mathrm{CL}$ on the $\frac{\sigma_{o b s}}{\sigma_{S M}}$ at $m_{H}=125 \mathrm{GeV}$ is observed to be 1.4 (1.3 expected), see Fig. $4 \mathrm{~b}$. The signal strength at $m_{H}=125 \mathrm{GeV}$ is found to be $\mu=0.2 \pm 0.5$ (stat.) \pm 0.4 (syst.). 


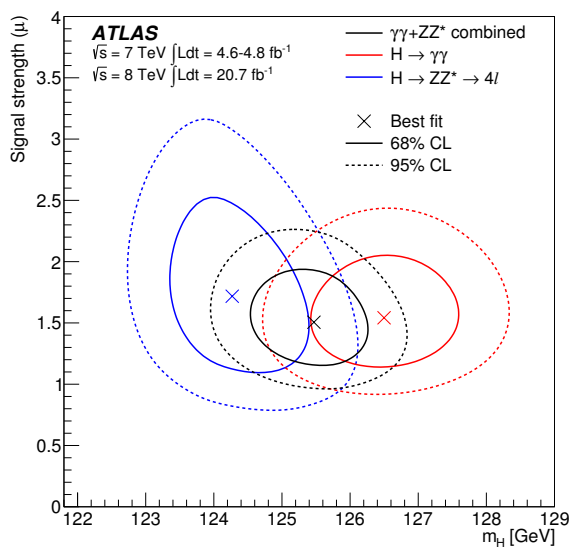

(a)

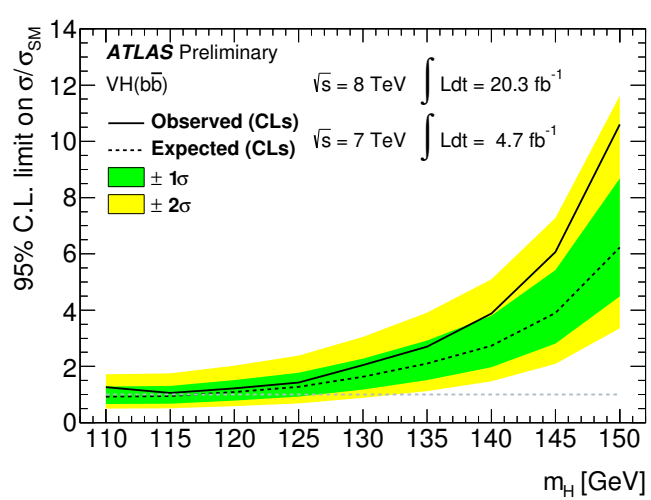

(b)

FIGURE 4. The signal strength as a function of $m_{H}$ for the $H \rightarrow \gamma \gamma$ and $H \rightarrow Z Z^{(*)} \rightarrow 4 l$ channels and their combination is shown in (a). The best-fit combined mass is $m_{H}=125.5 \pm 0.2$ (stat.) ${ }_{-0.6}^{+0.5}$ (syst.) $\mathrm{GeV}$ [13]. The plot in (b) shows the exclusion plot for the combined $V H \rightarrow V b \bar{b}$ analysis, where a 95\% CL is placed on $\frac{\sigma_{o b s}}{\sigma_{S M}}$ at $m_{H}=125 \mathrm{GeV}$ of 1.4 (1.3 expected)[22].

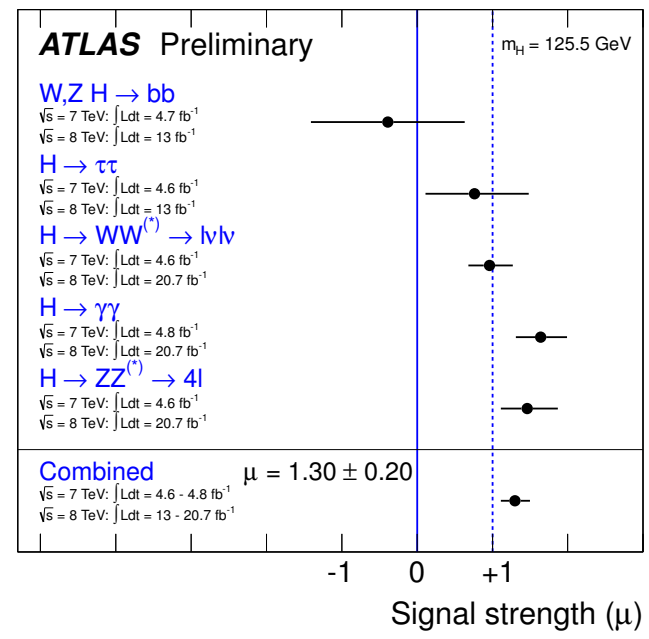

FIGURE 5. The signal strengths listed for each of the five channels, $H \rightarrow \gamma \gamma, H \rightarrow Z Z^{(*)} \rightarrow 4 l, H \rightarrow W W \rightarrow l v l v, H \rightarrow b \bar{b}$ and $H \rightarrow \tau^{+} \tau^{-}$, and their combined signal strength[10]. The combined signal strength is $1.3 \pm 0.2$.

\subsection{Combined Signal Strength Results}

The combined signal strength of all of the channels presented so far is calculated for a mass of $m_{H}=125.5 \mathrm{GeV}$, see Fig. 5. The signal strength is $\mu=1.3 \pm 0.2$ for the combination of all channels and all production modes. This is similar to the signal strength seen for the diboson channels separated from the fermionic decay channels, where it is found to be $\mu_{\text {diboson }}=1.33_{-0.18}^{+0.21}$ [13]. The consistency between the observed $\mu$ and the Standard Model expection of 1 is $7 \%$, which is increased to $14 \%$ using slightly more conservative uncertainties.

\section{SPIN AND PARITY MEASUREMENTS}

The spin of the Higgs boson has been measured by looking at the diboson decay channels. Kinematic observables of the final state particles give important information about the spin. The results of the spin analyses of the $H \rightarrow \gamma \gamma$, 


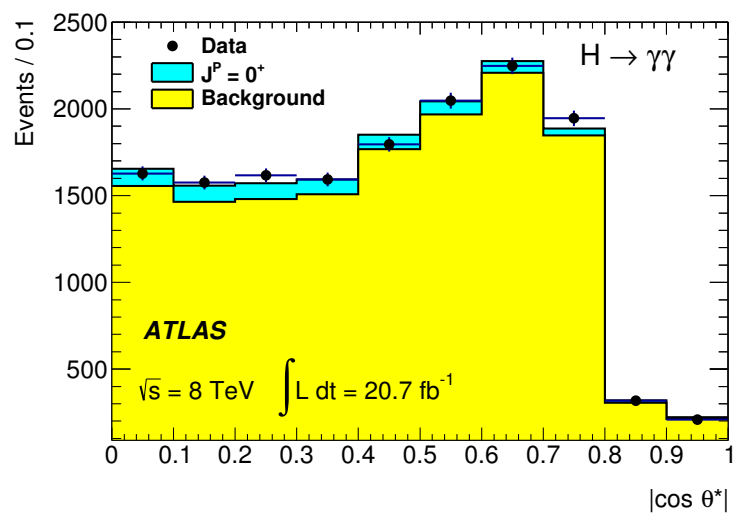

(a)

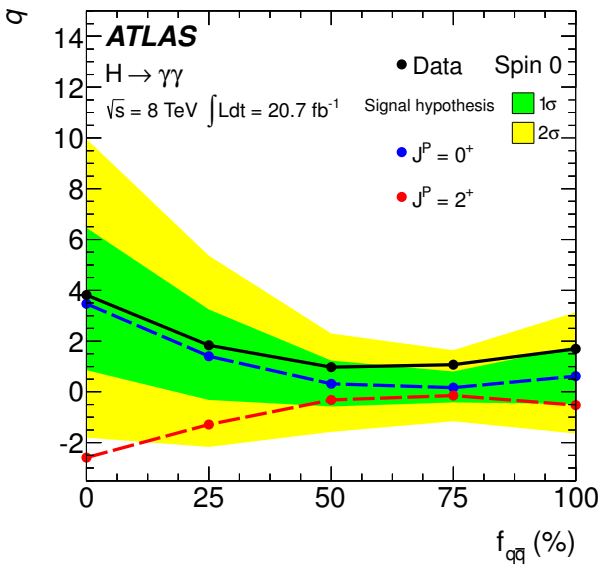

(b)

FIGURE 6. The distribution of $\left|\cos \theta^{*}\right|$, where $\theta^{*}$ is the polar angle of the photon with respect to the Collins-Soper frame, is shown in (a)[11]. The $J^{C P}=0^{+}$hypothesis shows good agreement with the data. The non-flat distribution is due to the background. The median expected values of the $\log$-likelihood ratio $q$ for the spin- 0 and spin- 2 hypotheses as a function of $f_{q \bar{q}}[11]$ are shown in (b).

$H \rightarrow Z Z^{(*)} \rightarrow 4 l$ and $H \rightarrow W W \rightarrow l v l v$ channels are discussed below. The $4 l$ analysis is also used to investigate the parity of the Higgs boson. The details of most of the signal discriminants, event categorisation and backgrounds have already been mentioned in the previous section. The spin and parity analyses are done using the full 2012 dataset at 8 $\mathrm{TeV}$, with the $4 l$ channel including the 2011 dataset at $7 \mathrm{TeV}$.

\section{1. $H \rightarrow \gamma \gamma$}

The $H \rightarrow \gamma \gamma$ decay mode is used to investigate the spin of the Higgs boson by using the invariant mass of the photon pair to identify Higgs candidates and the angular distribution of the photons in the resonance rest frame [23, 11] to measure the spin. The decay of a spin-1 particle into a photon pair is forbidden by the Landau-Yang theorem [14, 15], which gives strong evidence against the Higgs boson having spin 1. The possibility of a specific spin-2 "graviton-like" model with minimal SM couplings [17] is compared with the predicted SM spin-0 model. The spin-2 model can have the Higgs boson produced via gluon fusion or quark-antiquark annihilation. The admixtures of these production modes are varied for the analysis.

The polar angle $\theta^{*}$ of the final state photons with respect to the Collins-Soper frame [16] is used to investigate the spin properties. Spin-0 and spin-2 particles will have different $\cos \theta^{*}$ distributions. This distribution is mostly in the forward and backward directions for spin-2, and would be flat for spin-0 without acceptance limits. Fig. 6a shows compatibility between the spin-0 hypothesis and the data, where the non-flat distribution is due to the background. A log-likelihood ratio of the spin-0 and spin-2 hypotheses is used to compare the expected and observed results, shown in Fig. 6b. The log-likelihood is given as a function of the admixture of $q \bar{q}$ annihilation for the Higgs boson production, $f_{q \bar{q}}=\sigma_{H}^{q \bar{q}} / \sigma_{H}^{\text {total }}$. The spin-2 hypothesis is excluded at up to 99\% CL, depending on $f_{q \bar{q}}$.

$$
\text { 5.2. } H \rightarrow Z Z^{(*)} \rightarrow 4 l
$$

The $H \rightarrow Z Z^{(*)} \rightarrow 4 l$ channel has good sensitivity to both the spin and parity of the Higgs boson. The masses of the $Z^{(*)}$ bosons, one of the production angles and four decay angles are used to produce spin and parity dependent distributions[18], shown in Fig. 7a. The parity in spin-0 models can be compared by looking at the decay angles of $\Phi, \theta_{1}, \theta_{2}$, since the production angle $\theta^{*}$ and decay angle $\Phi_{1}$ are uniformly distributed. However, all of these angles are important for non-zero integer spins. In addition, the invariant masses of the leptonic decay pairs, $m_{12}$ and $m_{34}$, are sensitive to the spin and parity. Six hypotheses have been tested for $J^{P}: 0^{-}, 0^{+}, 1^{+}, 1^{-}, 2^{+}, 2^{-}$. The graviton-like $2^{+}$ 


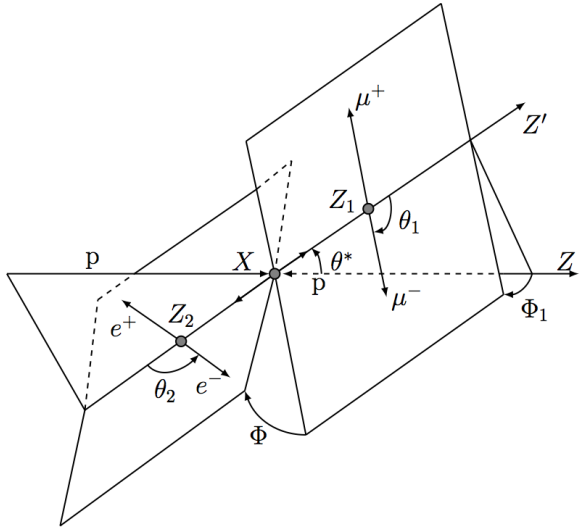

(a)

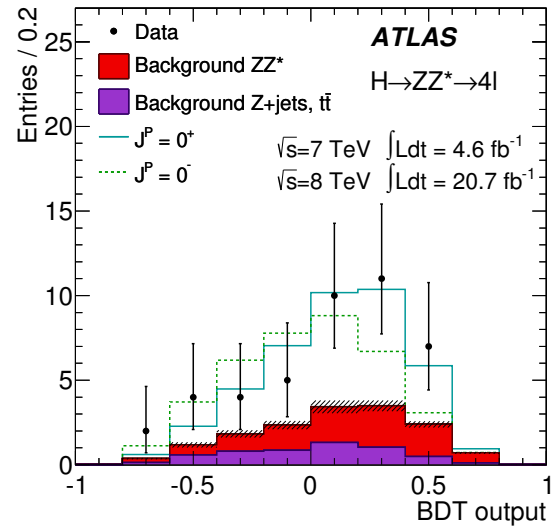

(b)

FIGURE 7. The definition of the production and decay angles for a $X \rightarrow Z Z^{(*)} \rightarrow 4 l$ decay[18] are shown in (b). $Z_{1}$ and $Z_{2}$ are in the rest frame of $X$, the beam axis is in the lab frame and the leptons in rest frames of $Z_{1}$ or $Z_{2}$. Plot (b) shows the BDT output for the $J^{C P}=0^{+}$and $J^{C P}=0^{-}$, from [11]. The positive parity hypothesis compares well with the observed data.

model [17] is equivalent to a Kaluza Klein graviton, and the $2^{-}$to a pseudo-tensor. Not all possible spin-2 models are investigated, however. Boosted Decision Trees (BDT) are trained and used to compare between different hypotheses (see Fig. 7b) and a matrix element likelihood method is used as a separate method.

The $0^{-}, 1^{+}$and $1^{-}$hypotheses have been excluded at $99.6 \%(97.8 \%), 99.4 \%(99.8 \%)$ and $96.9 \%$ (94.0\%) CLs, respectively, using the matrix element likelihood (BDT) method. The $0^{+}$is more compatible with a Higgs boson when compared with a graviton inspired $2^{+}$with minimal couplings to SM.

$$
\text { 5.3. } H \rightarrow W W^{(*)} \rightarrow l v l v
$$

The $H \rightarrow W W^{(*)} \rightarrow l v l v$ spin analysis uses the most sensitive subchannel $e v \mu v$. Positive parity is assumed, with the $0^{-}$and $1^{ \pm}$hypotheses already excluded by the $4 l$ analysis. A specific $2^{+}$model is compared with the SM expectation of $0^{+}$. The same angular decay characteristics used for the $H \rightarrow Z Z^{(*)} \rightarrow 4 l$ analysis are used, in addition to momenta measurements sensitive to spin. BDTs are trained on simulated $0^{+}$and $2^{+}$data and used to discriminate between them on the observed data. This is done separately depending on the value of $f_{q \bar{q}}$. The BDT output for $0^{+}$is shown in Fig. $8 \mathrm{a}$. The two spin models for varying admixtures of $f_{q \bar{q}}$ showing compatibility with the $0^{+}$model are showin in Fig. $8 b$.

\subsection{Combined Results}

The latest combined results are published in Ref. [11]. All of the diboson channels are combined to discriminate between the $0^{+}$and $2^{+}$models, where the $2^{+}$model is a graviton inspired model. The $2^{+}$model is excluded at $99.9 \%$ CL, independent of the fraction of gluon fusion or $q \bar{q}$ production (see Fig. 9).

\section{VBF AND COUPLINGS MEASUREMENTS}

The results of the measurements of the contribution of vector boson fusion production and the couplings of the Higgs boson to fermions and bosons are presented in this section. The event categories described for the different analyses in Section 4 allow for increased sensitivity to the different production mechanisms and Higgs boson couplings. These 


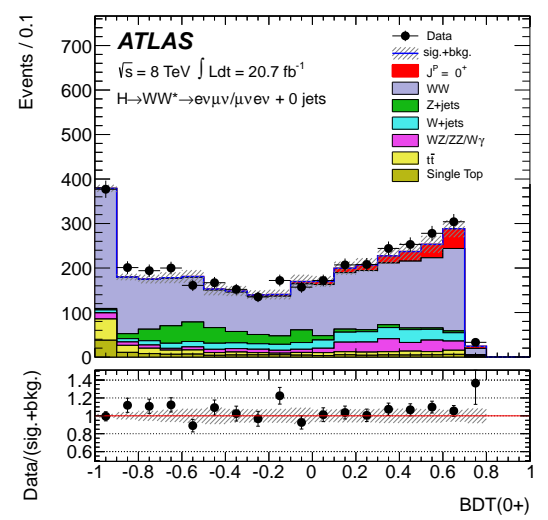

(a)

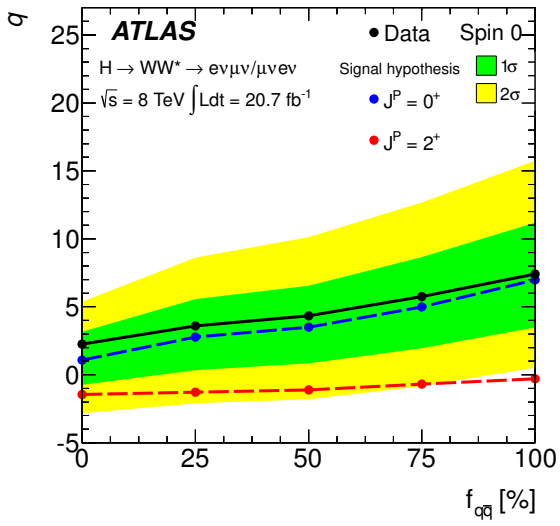

(b)

FIGURE 8. Distributions of the BDT output in the signal region are shown in (a). The distribution obtained in data is compared to the expected backgrounds and $0^{+}$signal hypothesis. BDT $\left(0^{+}\right)$refers to the BDT optimised for $0^{+}$separation from the background[11]. Observed values of the $\log$ likelihood ratio $q$ of the two spin hypotheses (solid line), as a function of the fraction of $q \bar{q}$ production of the spin-2 state[11] are shown in (b). The dashed lines show the expected spin- 0 and spin- 2 signals. The shaded light and dark bands correspond to one and two standard deviations around the spin-0 median curve, respectively.

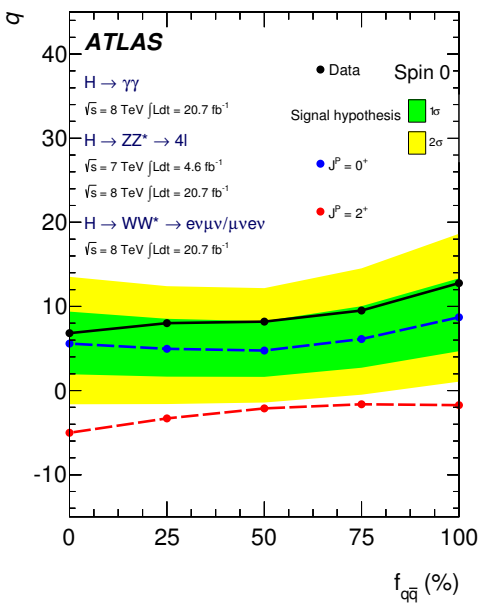

(a)

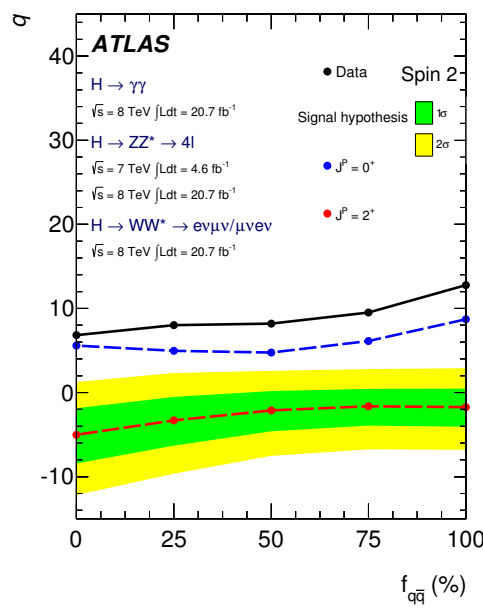

(b)

FIGURE 9. Distributions of the test statistic $q$, which is a log-likelihood ratio of the two spin hypotheses, for the combination of all diboson channels as a function of $f_{q \bar{q}}$, the fraction of $q \bar{q}$ production for the spin- 2 model. The green and yellow bands represent, respectively, the one and two standard deviation bands for the $0^{+}$(a) and for the $2^{+}$(b) hypotheses[11].

results are obtained using the diboson decay modes and the full 2011 and 2012 datasets at 7 and 8 TeV, respectively ${ }^{7}$. The latest published results can be found in Ref. [13].

\subsection{Evidence for Vector Boson Fusion Production}

The signal strengths shown in Section 4 do not offer the relative production mechanism contributions, however, using the event categorisation that separates the ggF, $V H$ and VBF production for the diboson decay channels this can

\footnotetext{
7 Only $13 \mathrm{fb}^{-1}$ used for the $\tau$ based analysis.
} 
be assessed in more detail. The ggF categories typically include contributions from the gluon mediated $t t H$ processes as well. The signal strength parameters $\mu_{\mathrm{ggF}+t t H}=\mu_{\mathrm{ggF}}=\mu_{t t H}$ and $\mu_{\mathrm{VBF}+V H}=\mu_{\mathrm{VBF}}=\mu_{V H}$ are used to measure the observed contributions relative to the Standard Model predictions (Fig. 10a). In order to combine the channels without assumptions on the SM branching ratios, the ratio $\left(\mu_{\mathrm{VBF}+V H} \times B / B_{\mathrm{SM}}\right) /\left(\mu_{\mathrm{ggF}}+t t H \times B / B_{\mathrm{SM}}\right)$ is used with a likelihood function $\Lambda\left(\mu_{\mathrm{VBF}+V H} / \mu_{\mathrm{ggF}+t t H}\right)$. The ratio $\mu_{\mathrm{VBF}} / \mu_{\mathrm{ggF}+t t H}$ is also used to test the $\mathrm{VBF}$ contribution, giving a result $\mu_{\mathrm{VBF}} / \mu_{\mathrm{ggF}+t t H}=1.4_{-0.3}^{+0.4}$ (stat. $)_{-0.4}^{+0.6}$ (syst.), which corresponds to $3.3 \sigma$ evidence for VBF production, which is reduced to $3.1 \sigma$ if the $H \rightarrow \tau^{+} \tau^{-}$channel is included.

\subsection{Couplings Measurements}

The couplings are measured using a leading order tree-level motivated framework with the following assumptions:

- A single signal resonance at $125.5 \mathrm{GeV}$ is assumed.

- The zero-width approximation is used where the predicted rate for a channel can be calculated using $\sigma \dot{B}(i \rightarrow$ $H \rightarrow f)=\frac{\sigma_{i} \dot{\Gamma}_{f}}{\Gamma_{H}}$ ), where $\sigma_{i}$ is the cross section for state $i, \Gamma_{f}$ is the partial width into the state $f$ and $\Gamma_{H}$ is the width of the Higgs boson.

- The observed state is a CP-even scalar.

Coupling scale factors, $\kappa_{j}$, are defined to measure the compatibility with the Standard Model of the observed cross section $\sigma_{j}$ and partial decay widths $\Gamma_{j}$ for particle $j$. These scale at $\kappa_{j}^{2}$ according to the SM prediction. The scale factor for $\Gamma_{H}$ is given by $\kappa_{H}$, which can then be combined with $\kappa_{j}$ for the particles in a Higgs production process to calculate the cross section. The full description of how these are defined can be found in [13]. Profile likelihood ratios of $\Lambda(\kappa)$ are used to create fits to the data and extract results. Ratios are defined between scale factors as $\lambda_{i j}=\kappa_{i} / \kappa_{j}$ to measure relationships between scale factors in some cases.

\subsubsection{Fermion and Vector Coupling}

To measure the couplings to fermions and vector bosons the coupling scale factors $\kappa_{F}$ and $\kappa_{V}$ are used, where all fermion couplings are assumed to be equal and all vector boson couplings are assumed to be equal. Two benchmarks are considered, the first assumes no contributions from beyond the Standard Model in the $H \rightarrow \gamma \gamma$ and $g g \rightarrow H$ loops and $\Gamma_{H}$ only depend on $\kappa_{F}$ and $\kappa_{V}$. Only $\kappa_{V}>0$ is assumed as the relative sign of the coupling scale factors is physical. The results are shown in Fig. 10b, with the $68 \%$ confidence levels obtained for each by profiling over the scale factor. Relaxing the constraint on no beyond the Standard Model physics $\lambda_{F V}$ and $\kappa_{V V}=\kappa_{V}^{2} / \kappa_{H}$ are introduced. The results of fits to these parameters give a $12 \%$ compatibility with the Standard Model and exclude vanishing couplings to fermions indirectly with a significance of $>5 \sigma$.

\subsubsection{Custodial Symmetry}

Custodial symmetry in the SM constrains the $W$ and $Z$ bosons to have the same couplings to the Higgs boson. This is tested by measuring the ratio $\lambda_{W Z}=\kappa_{W} / \kappa_{Z}$. The ratio of the branching ratios for $H \rightarrow W W^{*}$ and $H \rightarrow Z Z^{*}$ is normalised to the SM values and the likelihood function $\Lambda\left(\lambda_{W Z}\right)$ is used to provide a fit to the data, profiling different signal strengths. This method gives $\lambda_{W Z}=0.81_{-0.15}^{+0.16}$. When including $V H$ and VBF production and the $H \rightarrow \gamma \gamma$ channel this measurement becomes more sensitive. The diphoton channel is decoupled to remove potential beyond the SM contributions. This gives $\lambda_{W Z}=0.82 \pm 0.15$ with a $20 \%$ compatibility of the best-fit value with the SM expectation.

\subsubsection{Loop Couplings}

Heavy particles predicted from Beyond the SM scenarios could contribute to $g g \rightarrow H$ production and $H \rightarrow \gamma \gamma$ decays. To test for these contributions the Higgs boson width is assumed to have only SM contributions and all coupling scale factors are set to 1 , in accordance with the SM predictions. Two scale factors $\kappa_{g}$ and $\kappa_{\gamma}$ are used to measure the 


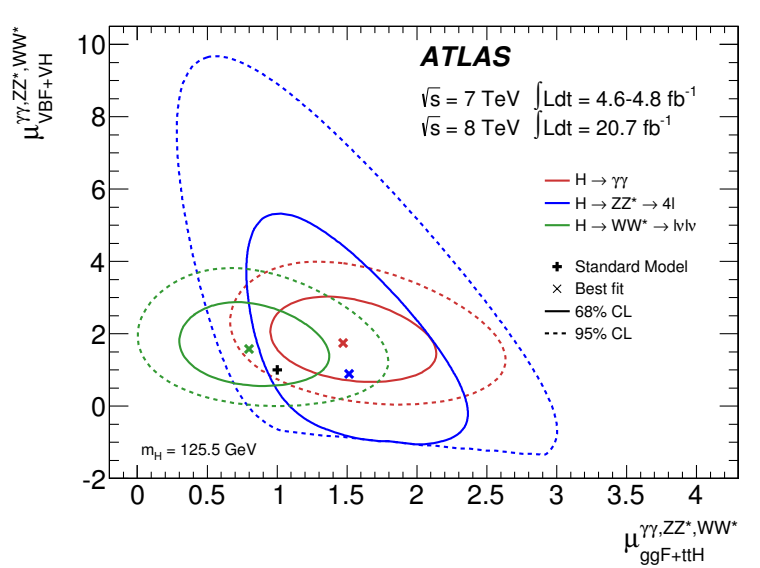

(a)

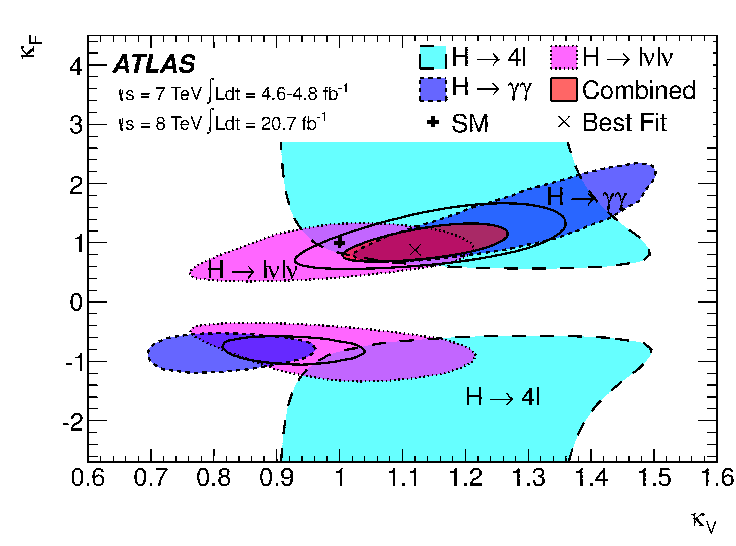

(b)

FIGURE 10. Plot (a) shows the likelihood contours for the Higgs boson diboson decay channels for $\mu_{\mathrm{VBF}+V H} \times B / B_{\mathrm{SM}}$ vs. $\mu \mathrm{ggF}+t t H \times B / B_{\mathrm{SM}}$ with $m_{H}=125.5 \mathrm{GeV}$. The branching ratio scale factors are included to remove dependence on the SM predictions. The dashed and solid lines represent $95 \%$ and $68 \%$ confidence levels, respectively, and the crosses show the best fit values. The SM prediction is shown as a + . Plot (b) shows the likelihood contours for the coupling scale factors of the Higgs boson to fermions and bosons, $\kappa_{F}$ and $\kappa_{V}$. The separate diboson search channels and their combination is plotted, along with their $68 \%$ confidence levels, and the Standard Model expectation. [13]

observed contributions to the loop induced processes. They are found to be $\kappa_{g}=1.04 \pm 0.14$ and $\kappa_{\gamma}=1.20 \pm 0.15$ (Fig. 11a), which has a compatibility between the best-fit value and the SM expectation of $14 \%$.

\subsubsection{Couplings Summary}

The results given here are based on the full 2011 and 2012 datasets for the three diboson channels, $H \rightarrow Z Z^{(*)} \rightarrow 4 l$, $H \rightarrow W W \rightarrow l v l v$ and $H \rightarrow \gamma \gamma$. Constraints on the couplings to vector bosons are placed at the $\pm 10 \%$ level. Further, there is a $>5 \sigma$ indirect evidence for fermion couplings. The ratio of the couplings to the $W$ and $Z$ bosons is consistent with the SM expectation of 1 . The analysis of the loop couplings shows no anomalous contributions to the gluon mediated processes, $g g \rightarrow H$ and $H \rightarrow \gamma \gamma$. The final coupling scale factors are shown in Fig. $11 \mathrm{~b}$.

\section{SUMMARY}

The updated results in the search for the SM Higgs boson and the measurements of its properties using the ATLAS detector are presented. Up to $25 \mathrm{fb}^{-1}$ of data at $\sqrt{s}=7 \mathrm{TeV}$ and $\sqrt{s}=8 \mathrm{TeV}$ have been analysed in the $H \rightarrow \gamma \gamma$, $H \rightarrow Z Z^{(*)} \rightarrow 4 l, H \rightarrow W W \rightarrow l v l v, H \rightarrow \tau^{+} \tau^{-}$and $H \rightarrow b \bar{b}$ decay modes. The latest mass measurements and signal strength are reported, with a combined mass and signal strength of $m_{H}=125.5 \pm 0.2$ (stat.) ${ }_{-0.6}^{+0.5}$ (syst.) $\mathrm{GeV}$ and $\mu=1.3 \pm 0.2$, respectively. The combined spin analysis shows very good evidence for a $J^{C P}=0^{+}$particle. Indirect couplings to fermions are found with $>5 \sigma$ significance, although confirmation through the $H \rightarrow \tau^{+} \tau^{-}$and $H \rightarrow b \bar{b}$ decay modes will be an important test. The couplings to fermions and bosons have been constrained and no anomalous contributions to loop-induced processes are reported. There is evidence at $3.3 \sigma$ significance for the VBF production mechanism. All of the measurements reported are consistent with the Standard Model Higgs boson.

\section{ACKNOWLEDGMENTS}

I would like to thank the ATLAS Collaboration for the opportunity to give this presentation, the University of Edinburgh and SUPA for the financial support, and Dr. Victoria Martin, Dr. Wahid Bhimji and Dr. Eric Feng for the 


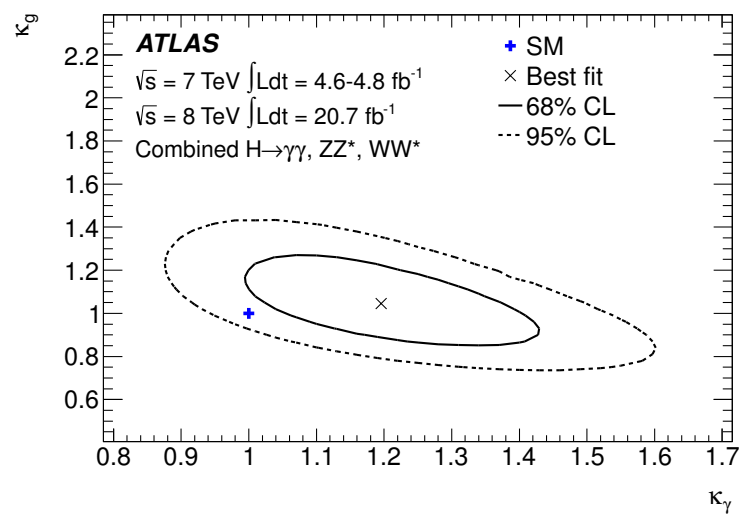

(a)

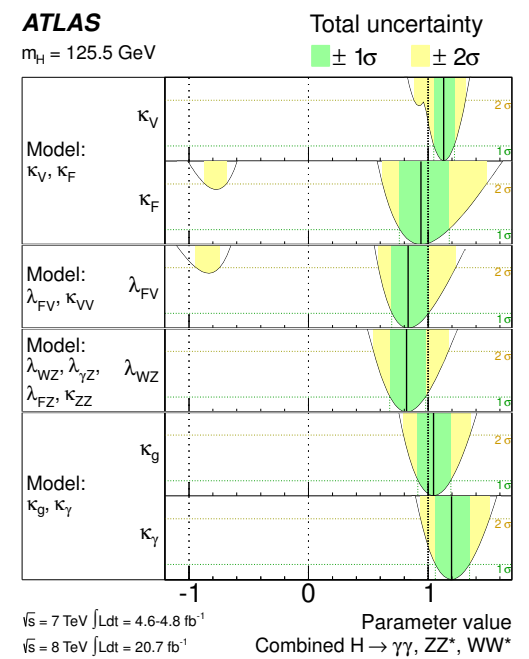

(b)

FIGURE 11. Plot (a) shows the likelihood contours and best-fit result for the coupling scale factors $\kappa_{\gamma}$ and $\kappa_{g}$ which are used to test for any BSM contributions to $H \rightarrow \gamma \gamma$ and $g g \rightarrow H$. Plot (b) shows the results for all the coupling scale factors for a Higgs boson of mass $m_{H}=125.5 \mathrm{GeV}$. The uncertainties are shown in the shaded bands and the best-fit values by a solid vertical line.[13]

helpful reviews and practice talks. I would like to thank the organisers of the IMFP 2013 conference for an excellent conference and the financial support I received from them, the University of Malaya and Instituk Fizik Malaysia.

\section{REFERENCES}

1. F. Englert and R. Brout, "Broken Symmetry and the Mass of Gauge Vector Mesons," Phys. Rev. Lett. 13 (1964) 321.

2. P. W. Higgs, "Broken symmetries, massless particles and gauge fields," Phys. Lett. 12 (1964) 132.

3. P. W. Higgs, "Broken Symmetries and the Masses of Gauge Bosons," Phys. Rev. Lett. 13 (1964) 508.

4. P. W. Higgs, "Spontaneous Symmetry Breakdown without Massless Bosons," Phys. Rev. 145 (1966) 1156.

5. ATLAS Collaboration, Phys.Lett. B716 (2012) 1-29, http: / / arxiv . org/abs/1207. 7214.

6. J. Baglio and A. Djouadi, JHEP 1103 (2011) 055, http: / / arxiv.org/abs/1012.0530.

7. LHC Higgs Cross Section Working Group and S. Dittmaier, C. Mariotti, G. Passarino and R. Tanaka (Eds.),Handbook of LHC Higgs Cross Sections: 1. Inclusive Observables, http: //arxiv.org/abs/1101.0593.

8. L. Evans and P. Bryant, "LHC Machine," JINST 3 (2008) S08001.

9. ATLAS Collaboration, 2008 JINST 3 S08003.

10. ATLAS Collaboration, ATLAS-CONF-2013-034, http://cds. cern. ch/record/1528170.

11. ATLAS Collaboration, CERN-PH-EP-2013-102, Submitted to Phys. Lett. B, http: //arxiv . org/abs/1307.1432.

12. ATLAS Collaboration, Phys. Rev. Lett. 108 (2012) 111803.

13. ATLAS Collaboration, Phys. Lett. B (2013), http://dx.doi.org/10.1016/j.phys letb.2013.08.010.

14. L. D. Landau, Dokl. Akad. Nauk Ser. Fiz. 60 (1948) 207-209.

15. C.-N. Yang, Phys. Rev. 77 (1950) 242-245.

16. J. C. Collins and D. E. Soper, Phys. Rev. D 16 (1977) 2219-2225.

17. Y. Gao, A. V. Gritsan, Z. Guo, K. Melnikov, M. Schulze, and N. V. Tran, Phys. Rev. D 81 (2010) 075022.

18. ATLAS Collaboration, ATLAS-CONF-2013-013, http://cds . cern. ch/record/1523699.

19. ATLAS Collaboration, ATLAS-CONF-2013-014, http://cds.cern. ch/record/1523727.

20. ATLAS Collaboration, ATLAS-CONF-2012-160, http://cds. cern. ch/record/1493624.

21. A. Elagin, P. Murat, A. Pranko, A. Safonov (2010), Nucl. Instrum. Meth. A 654, (2011) 481-489.

22. ATLAS Collaboration, ATLAS-CONF-2013-079, http://cds. cern. ch/record/1563235.

23. ATLAS Collaboration, ATLAS-CONF-2013-029, http://cds. cern. ch/record/1527124.

24. ATLAS Collaboration, ATLAS-CONF-2013-031, http://cds.cern. ch/record/1527127.

25. ATLAS Collaboration, ATLAS-CONF-2013-012, http://cds. cern. ch/record/1523698.

26. ATLAS Collaboration, ATLAS-CONF-2013-030, http://cds. cern. ch/record/1527126. 